\title{
Scolia
}

Revue de linguistique

Espace et temps : quelles asymétries ?

\section{Gilles CoL, Construction du sens : un modèle instructionnel pour la sémantique}

\section{Graham Ranger}

\section{OpenEdition}

\section{Journals}

Édition électronique

URL : http://journals.openedition.org/scolia/1011

DOI : 10.4000/scolia.1011

ISSN : 2677-4224

\section{Éditeur}

Presses universitaires de Strasbourg

\section{Édition imprimée}

Date de publication : 11 juillet 2019

Pagination : 175-179

ISBN : 979-1-03440-050-8

ISSN : 1253-9708

Référence électronique

Graham Ranger, "Gilles CoL, Construction du sens : un modèle instructionnel pour la sémantique », Scolia [En ligne], 33 | 2019, mis en ligne le 11 juillet 2019, consulté le 25 septembre 2020. URL : http:// journals.openedition.org/scolia/1011 ; DOI : https://doi.org/10.4000/scolia.1011 
Gilles COL, Construction du sens: un modèle instructionnel pour la sémantique, Bruxelles, Peter Lang, collection Linguistic Insights. Studies in Language and Communication, vol. 224, 2017, 294 pages.

Le livre de Gilles Col, Construction du sens: un modèle instructionnel pour la sémantique, constitue, d'après la quatrième de couverture, "une première tentative de modélisation du processus de construction du sens dans son déroulement progressif et dynamique». Ce défi est relevé avec beaucoup de bonheur au cours d'un travail passionnant qui réussit à faire dialoguer différentes approches linguistiques dans une démarche toujours constructive, comme nous le verrons ci-dessous.

L'ouvrage est structuré en trois grandes parties, obéissant à une présentation progressive des problématiques et à des perspectives de réponse données par l'auteur. La première partie parcourt en effet l'état de l'art sur la question de la polysémie, avant de proposer une modélisation d'items linguistiques en termes abstraits de " convocation / évocation" en interaction avec une "scène verbale». La deuxième partie fournit plusieurs illustrations de la méthode, essentiellement à partir de quelques marqueurs de l'anglais (over, by, until, on). La troisième partie considère la modélisation des relations entre marqueurs linguistiques dans une construction dynamique du sens s'opérant au fur et à mesure qu'on avance dans l'énoncé. Une conclusion récapitulative reprend les grandes lignes de l'argumentation.

La présente recension considérera chaque partie avant de proposer une évaluation critique du travail de l'auteur.

Après une brève introduction, où l'auteur présente ses objectifs ainsi que sa démarche pluridisciplinaire, la première partie considère le problème de la polysémie en langue naturelle. Col s'oppose à l'inévitable prolifération des significations qu'engendrent le traitement par l'homonymie et, dans une moindre mesure, la sémantique du prototype. À partir des cas de l'adjectif blanc en français ou du verbe appear en anglais, Col montre comment les proximités entre significations se prêtent à une représentation informatisée, sous forme de graphe de synonymie. La finalité de ce type de schéma se limite cependant à de la représentation, saisissante peut-être, mais sans vertu explicative a priori. L'explication, pour Col, va de pair avec la reconnaissance de l'interdépendance des unités dans une compositionnalité gestaltiste et évolutive qui rend compte de la construction du sens «dans le déroulement même de l'énoncé» (p. 49). 
Le deuxième chapitre présente le concept d' "instruction», que l'auteur compare successivement à des notions similaires dans la théorie de l'optimalité, chez Anscombre et Ducrot (1983), Nølke (1994) ou Némo (2001). Col rapproche sa conception de l'instruction aux espaces mentaux de Fauconnier (1984), ou à la forme schématique de Culioli (1990), en précisant qu'à la différence de ces modèles, l'instruction doit prévoir «dans sa formulation son assemblage avec une autre forme» (p. 63). Le modèle de la construction du sens présenté s'inspire de la psychologie cognitive d'un Barsalou (par ex. 1999), en ce que «le sens d'un énoncé est une série de représentations modifiées au fur et à mesure du traitement» (p. 58). Ce chapitre se termine par une série de définitions de concepts opératoires dans le modèle de Col: «scène verbale», «fenêtrage», "saillance», etc.

Une fois effectuée la présentation générale du modèle, la deuxième partie du livre se focalise sur des études de cas précis. L'élaboration d'une «instruction» passe pour Col par «l'étude de l'unité non pas comme une unité isolée mais prise dans un réseau complexe et prototypique d'unités co-textuelles" (p. 107). Cette démarche va de pair avec une méthodologie basée sur corpus, suivant en cela les travaux de Hanks (2004): «l'instruction de construction du sens [...] contribue au sens de l'énoncé $[\ldots]$ mais en retour, elle s'enrichit du sens du contexte et de l'énoncé qu'elle contribue à construire» (p. 108). À la suite d'un compte rendu critique des travaux antérieurs, Col propose une analyse du marqueur de l'anglais over, porteur, d'après l'auteur, de l'instruction suivante: "OVER convoque un domaine borné; OVER évoque un mouvement de recouvrement du domaine, frontières incluses». (p. 135). Le modèle ainsi posé est appliqué à la diversité d'emplois de over (Go over the bridge, Her romance... is over, The process has evolved over the decade, etc.). Le modèle défendu s'oppose ainsi à la primauté d'un sens spatial pour le marqueur over, approche souvent défendue dans les théories cognitivistes, au bénéfice d'une instruction plus abstraite, compatible avec les différents emplois contextualisés, au terme de l'interaction avec le co-texte. Il est également à noter que le modèle ne prévoit pas de catégorie grammaticale par avance (préposition, particule, adverbe, etc.), celleci étant également construite en interaction avec le contexte. Nous ne nous attarderons pas sur les chapitres 4 et 5 , plus synthétiques, qui considèrent dans la même optique les marqueurs by et until, puis on. 
La troisième partie est consacrée au traitement des instructions les unes par rapport aux autres, dans les énoncés. Le chapitre 6 présente de manière relativement décontextualisée, un «répertoire instructionnel pour l'anglais», où sont proposées les instructions pour un nombre limité de marqueurs de l'anglais, jouant au niveau des «entités» de la scène verbale (les articles, this et that, some et any, les pronoms), de ses "procès» (temps et aspects), de sa "topologie» (of, off, out, always, still, already). Le chapitre suivant s'adresse au problème critique de «l'ordre de traitement des unités» (p. 209). En effet, étant donné les instructions pour chaque élément dans un énoncé donné, dans quel ordre ces instructions doivent-elles s'appliquer? En complément de l'ordre linéaire d'apparition des termes, Col note la possibilité pour la construction du sens de certains items d'être "mise en attente» (p. 211) avant traitement. Les apports de la psychologie cognitive (Tomlin, 1997, par exemple) sont pris en compte dans une discussion sur la signification de la position initiale, en particulier. On aboutit à un modèle qui évoque "une série de représentations modifiées au fur et à mesure du traitement» (p. 220) dans un mouvement de va-et-vient évolutif dans la construction d'un sens qui, s'il n'est pas total, est au moins suffisant pour ne pas être bloquant. Le huitième et dernier chapitre met à l'épreuve le modèle à partir d'un travail incrémental sur la construction du sens dans les énoncés. Plusieurs cas sont considérés, en anglais et en français, afin d'exposer, par l'exemple, le processus de va-et-vient, le principe de la «mise en attente», etc. à l'œuvre dans la construction du sens. Au cours du chapitre, Col revient sur le modèle proposé pour over, en indiquant désormais plus précisément la manière dont le sens et, partant, la catégorie de cet item se précisent en contexte. Le rôle de la prosodie fait également l'objet d'une considération particulière. Pour ne donner qu'un exemple de la méthode de construction du sens incrémentale prônée dans l'ouvrage, l'ordre de traitement des huit éléments de l'énoncé J'ai vu un tableau dans la pièce est, d'après Col, J'(1) voir (4) passé composé (5), un (3), tableau (2), dans (8), la (7), pièce (6). Ainsi le sens de certains items est mis en attente des déterminations complémentaires apportées par la suite. Pour savoir comment interpréter voir il nous faut connaître sur quoi il porte, pour interpréter dans il nous faut attendre les déterminations apportées par la pièce, etc. 
L'ouvrage de Col représente une contribution majeure à la théorie de opérations prédicatives et énonciatives, que l'auteur réussit, avec pédagogie et érudition, à faire entrer en dialogue avec d'autres théories, d'autres méthodologies. Nous avons apprécié en particulier l'association des données quantitatives à une modélisation de l'activité langagière, voie qui nous paraît particulièrement prometteuse pour les recherches à venir, autant du côté du traitement automatique du langage que du côté énonciativiste. On salue également l'ambition innovatrice de la tentative de modélisation de la construction incrémentale du sens dans une démarche compositionnelle, gestaltiste, qui ne perd jamais de vue le caractère inévitablement séquentiel du langage et, partant, de la construction du sens qui s'y opère.

On pourrait néanmoins formuler quelques réserves, d'abord sur l'utilisation faite des corpus dans le travail de Col. La conclusion se réclame d'une démarche qui serait "corpus-driven", l'introduction évoque plutôt une démarche "corpus-based", en définitive, le caractère déterminant du corpus dans l'élaboration et la mise à l'épreuve des hypothèses n'est pas visible au même degré dans tous les chapitres et parait nettement plus réussi dans le chapitre 3, consacré à over. Dans la mesure où les différents chapitres de l'ouvrage sont partiellement issus de travaux antérieurs, on peut supposer que le traitement variable des données issues des corpus est le reflet partiel de l'évolution méthodologique de l'auteur. On regrette un certain nombre de scories formelles (coquilles, numérotation d'exemples ou de figures, référencement non homogène des exemples), qui seront sans doute éliminées dans une deuxième édition. Enfin, il paraît dommage, du point de vue de la diffusion des thèses que véhicule l'ouvrage, que l'auteur ait si souvent privilégié des marqueurs anglais. Pour atteindre un public plus important, il serait plus logique d'associer la langue de rédaction et donc du lectorat visé, et les marqueurs étudiés.

Ces quelques réserves n'enlèvent rien à l'appréciation très largement positive que nous formulons sur l'ouvrage de Gilles Col, dont l'ambition et la pédagogie sont à saluer. Nous ne doutons pas que ce travail marquera un jalon incontournable dans l'évolution des théories énonciativistes et, plus généralement, dans la modélisation de l'activité langagière. 


\section{Références}

ANSCOMBRE J.-C. \& DUCROT O. (1983), L'Argumentation dans la langue, Bruxelles, Mardaga.

BARSALOU L. W. (1999), Perceptual Symbol Systems, Behavioral and brain sciences 22, 577-660.

CULIOLI A. (1990), Pour une linguistique de l'énonciation, vol. 1, Paris, Ophrys.

FAUCONNIER G. (1984), Espaces mentaux. Aspects de la construction $d u$ sens dans les langues naturelles, Paris, Éditions de Minuit.

HANKS P. (2004), Corpus Pattern Analysis, in Williams G. \& Vessier S. (eds.), Proceedings of the 11th EURALEX International Congress, Lorient, Université de Bretagne-Sud, Faculté des Lettres et des Sciences Humaines, 87-97.

NÉMO F. (2001), Pour une approche indexicale (et non procédurale) des instructions sémantiques, Revue de Sémantique et de Pragmatique 9-10, 195-218.

NØLKE H. (1994), Linguistique modulaire: de la forme au sens, Louvain La Neuve, Peeters.

TOMLIN R. (1997), Mapping Conceptual Representations into Linguistic Representations: The Role of Attention in Grammar, in Nuyts J. \& Pederson E. (eds.), Language and Conceptualization, Cambridge, Cambridge University Press, 162-189. 